\title{
BUILDING BETTER TOGETHER: INTERPROFESSIONAL REFLECTIONS ON DESIGNING ASSISTIVE TECHNOLOGY
}

\author{
Claire Davies, Elizabeth Delarosa, Susanne Murphy, Catherine Donnelly \\ Queen’s University, Kingston Ontario \\ claire.davies@queensu.ca
}

\begin{abstract}
This paper presents the results of a student questionnaire after completion of the course "Building Better Together (BBT)". BBT is an interdisciplinary project-based teaching and learning experience that facilitated a collaboration between the engineering and occupational therapy programs within Queen's University. This project integrated different frameworks to develop competencies for students from both programs which included: an engineering design process, a clinical process framework used by occupational therapists, and an interprofessional framework. We share the responses from an inter-professional questionnaires that included feedback about discipline-specific learning objectives, resources utilized, weekly course format, and outcomes of this experience.
\end{abstract}

Keywords: Interdisciplinary, education, design, assistive devices, occupational therapy, engineering

\section{INTRODUCTION}

Studies have found that between $18-53 \%$ of assistive technology prescribed to older adults is not used [1]. These findings reflect the critical importance of understanding the complex interplay between personal factors and the technology. We sought to train students in biomechanical engineering and occupational therapy (OT) in a course designed to meet the actual needs of a community end-user. To evaluate the perceived benefits from this collaboration, two interprofessional surveys were conducted.

\subsection{Teaching approach}

Building better together (BBT) provided students a complex real-life problem that would benefit from perspectives, knowledge, and skills from different disciplines. The project merged the lived experience of older community members with clinical knowledge of disability and expertise in design. The overall outcome provided a model to engage in designing a novel assistive device with an older adult that would enable them to perform an activity that they reported as being challenging.

Approximately 130 students from both OT and Engineering were allocated to 24 design teams. Design teams were made up of at least three occupational therapy students and 2-3 engineering students. Each design team was partnered with an older adult. The older adults who participated in this project were living in the Kingston community with varying abilities and impairments including muscular dystrophy, neuropathy, Parkinson's disease, hip fracture, and an older caregiver/husband.

\subsection{Canadian Interprofessional Health Collaborative Framework}

In BBT the Canadian Interprofessional Health Collaborative (CIHC) framework was applied within an academic environment. The CIHC framework was developed to inform education and practice across health professions based on a standard approach to competencies [2]. Engineering and OT students worked together using an an interdisciplinary approach to design a device to meet the needs of a specific community end-user. The CIHC framework was used to guide shared curriculum for this project to facilitate the development of transferable skills, such as teamwork, conflict resolution, and communication. Specific skills were the focus of individual tutorials; in the initial orientation, design teams were asked to establish a team contract and discuss roles and responsibilities, for example. Most of the inter-professional skills were facilitated during natural teaching moments, where design teams had to learn to effectively work together and problem-solve.

\subsection{Objectives}

To better understand the team collaborative environment, this part of the project involved understanding the student experience. We sought to:

1) evaluate the teaching offerings from a quality assurance perspective

2) survey students about the perceived effectiveness of learning in interdisciplinary teams. 


\section{METHOD}

\subsection{Teaching approach}

One hour per week included a joint lecture taught by an engineering instructor. All other lectures were taught independently by members of the home department. Once a week for two hours, the 24 teams of three occupational therapists and three engineers would come together to design a device for a community end-user. Joint assignments included development of a prototpye using the parallel design process, and a poster presentation at the end of the course. In addition, each had three additional assignments that were focused on discipline specific learning attributes. Two interprofessional surveys were emailed to the students upon completion of the course.

\subsection{Questionnaires to Evaluate Interdisciplinary collaboration}

Two questionnaires were used to evaluate the interdisciplinary collaborations within the teams. These included the:

a) Online Professional Roles Knowledge (OPRK) questionnaire: An online questionnaire developed to assess student's knowledge about the other profession. No similar questionnaires have been found in the literature. The questionnaire will included both open-ended and close-ended question and was administered before and after the course.

b) Readiness of Health Care Students for Interprofessional Learning (RIPLS) is a questionnaire designed to assess students' readiness to learn collaboratively with other health care students. The RIPLS was modified to consider the non-health care context/discipline and will be administered before and after the course.

\section{RESULTS}

Of the 128 students registered in the course, 58 completed the OPRK survey and 51 completed the RIPLS survey. Thirty percent indicated that they had previously worked within interdisciplinary teams, and $88 \%$ believed that by learning how to interact within an interdisciplinary team, they would be more effective. All agreed or strongly agreed that patients would benefit from an interdisciplinary team approach.

\subsection{Teaching Strategies}

The majority, 84\%, agreed that the purpose and learning objectives of the course were made clear. However, only $51 \%$ of the students felt that they had received sufficient orientation to the project.

When asked about whether the teaching strategies helped their learning, $46 \%$ agreed or strongly agreed while
$34 \%$ disagreed. It was suggested that $56 \%$ of the students would prefer the instructors to be of the same discipline as the students (Figure 1), though 80\% agreed that they valued having more than their own discipline involved in the teaching.

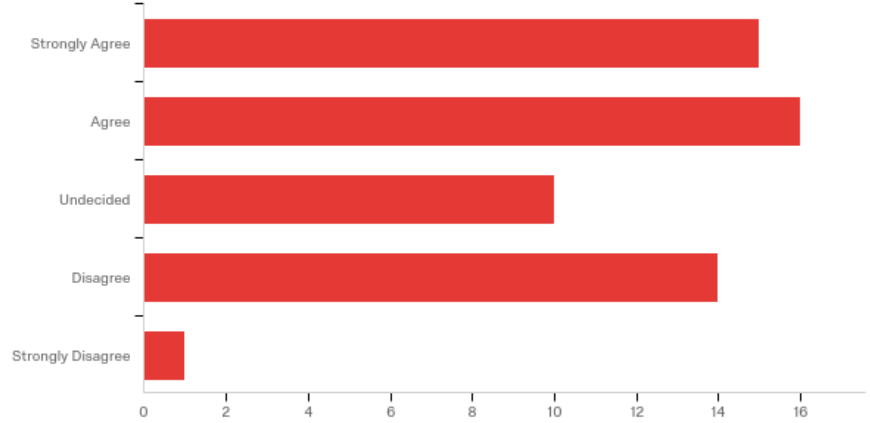

Fig. 1. Response distribution when asked "My preference is for teachers to be the same discipline as the student" (strongly agree at the top and strongly disagree at the bottom)

\subsection{Perceived effectiveness of learning opportunities}

From an overall perspective, $75 \%$ felt they had sufficient clinical learning opportunities, and $70 \%$ indicated that sufficient technical learning opportunities were explored. They were also convinced that the project had given them new insights on how to effectively design (85\%), with $88 \%$ saying they had a better understanding of their role in an interprofessional team, and $91 \%$ suggesting they better understood the roles and functions of other disciplines in service delivery.

\subsection{Qualitative reflections}

When given the opportunity to comment about the interdisciplinary learning approach, one stated:

"A lot of it was an unnecessary wasted time especially for an engineering student with a great deal of other responsibilities to take care of. The design process would be significantly more efficient as a student alone, without the inclusion of occupational therapy students. They just don't think and understand to the same degree."

while another suggested:

"I really liked the course, and got a lot out of it! (How to discuss things I know really well, that other team members don't)".

\section{DISCUSSION}

The distribution of results was bimodal for several of the questions. Unfortunately, we did not ask the discipline of the student prior as the first question in the survey. It is possible, that because the professor teaching joint portion of the course was an engineer and the teaching assistants were engineers, that the occupational therapists did not feel that their needs were adequately addressed. It is interesting 
that half believed that the instructors should be from their own discipline, yet $80 \%$ also saw the value in having both disciplines involved in the teaching. This suggests that interdisciplinary teaching is valued by the students. It would be valuable to ensure a balance of teaching time among the two disciplines. This was attempted using a blended learning technique in which the occupational therapists were provided video guides about the engineering tools and the engineers were provided videos about the occupational therapy tools. However, during this course offering, it was not a requirement to watch these videos. In the future, blended learning through video analysis should be required for both disciplines.

While there was a bimodal distribution on teaching and learning preferences, there was convincing support for the course with respect to technical and clinical experiences. Students realized the importance of working together on interdisciplinary teams and gained knowledge that will benefit them when working with others.

\section{CONCLUSION}

Teaching and learning within interdisciplinary teams increased awareness, knowledge and communication skills. It is important to ensure a balanced approach to the teaching by ensuring equal distribution among the engineering and occupational therapy teaching faculty.

\section{Acknowledgements}

We would like to thank the Centre for Teaching and Learning and the Faculty of Engineering and Applied Science at Queen's University for providing financial support to develop Building Better Together (BBT). The Social Sciences and Humanities Research Council (SSHRC) has also provided funding (430-2017-00956) to evaluate the outcomes of this method of teaching.

Additionally, we wish to thank all the students who participated as part of their coursework and the teaching assistants, Elizabeth Hoskin, Rhianne Lopez, and Alannah Lax-Vanek for all their assistance with making the course proceed smoothly.

\section{References}

[1] E. M. Agree and V. A. Freedman, "A comparison of assistive technology and personal care in alleviating disability and unmet need," Gerontologist, vol. 43, pp. 335-44, Jun 2003.

[2] V. Wood, A. Flavell, D. Vanstolk, L. Bainbridge, and L. Nasmith, "The road to collaboration: developing an interprofessional competency framework," J Interprof Care, vol. 23, pp. 621-9, Nov 2009. 